\title{
BN-C Hybrid Nanoribbons as Gas Sensors
}

\author{
MAHDI DARVISHI GILAN ${ }^{1}$ and RAAD CHEGEL (1) ${ }^{1,2}$ \\ 1.-Physics Department, Faculty of Science, Malayer University, Malayer, Iran. 2.-e-mail: \\ raad.chegel@gmail.com
}

\begin{abstract}
The effects of carbon monoxide $(\mathrm{CO})$ and ammonia $\left(\mathrm{NH}_{3}\right)$ molecules adsorption on the various composites of boron nitride and graphene $\mathrm{BN}-\mathrm{C}$ hybrid nanoribbons are investigated using the non-equilibrium Green's function (NEGF) technique based on density functional theory (DFT). The effects of adsorption with possible random configurations on the average of the density of states (DOS), transmission coefficient, and the current-voltage $(I-V)$ characteristics are calculated. The results indicate that, by embedding armchair graphene nanoribbon (AGNR) with boron nitride nanoribbon (BNNR), the various electronic properties can be observed after gas molecule adsorption. The electronic structure and gap of hybrids system is modified due to gas adsorption, and the systems act like the $n$-type semiconductor by $\mathrm{NH}_{3}$ molecule adsorption. The hybrid structures due to their tunable band gap are better candidates for gas detecting compared to the pristine BNNRs and AGNRs.
\end{abstract}

Key words: BN-C hybrid nanoribbons, gas sensor, non-equilibrium Green's function, transfer matrix

\section{INTRODUCTION}

The synthesis of carbon nanotubes (CNTs), graphene and another materials such as $\mathrm{BN}$ and $\mathrm{SiC}$ with a wide band gap have stimulated numerous research investigations according to their novel and potential applications in the electronic and optoelectronic devices on a nanometer scale. ${ }^{1}$ Due to their high surface-area-to-volume ratio, the electronic and optical properties of these materials can be influenced by adding gas molecules. All these properties make them as an attractive candidate for sensing various molecules.

The nanostructures cannot detect some of molecules that are weakly adsorbed on their surfaces. The CNT sensor devices could be used to detect small concentrations of $\mathrm{NH}_{3}, \mathrm{NO}_{2}$, and $\mathrm{O}_{2}$ while toxic gases such as $\mathrm{CO}$ cannot be detected using these devices. ${ }^{2}$ Unlike the pristine CNTs, the CO can only be physisorbed on the sidewall of BNNT through van der Waals interactions. ${ }^{3}$

(Received May 20, 2017; accepted October 6, 2017;

published online October 30, 2017)
The defects such as Stone-Wales, vacancies, and doping can be improved against the limitations of gas sensing in these structures. ${ }^{4-8}$

Investigation of the electronic properties of the Cdoped BNNT in the presence of CO molecules by first-principle calculations, showed that the interaction of $\mathrm{CO}$ molecules can be enhanced in C-doped BNNTs in comparison with pure BNNT. ${ }^{4}$

The adsorption of $\mathrm{CO}$ molecule on transition metals (TM)-doped BNNTs leads to different electronic properties and can be used as a $\mathrm{CO}$ gas sensor. ${ }^{9}$ The $\mathrm{NH}_{3}$ molecule in a defected $\mathrm{BN}$ sheet has more negative adsorption energy rather than a pristine $\mathrm{BN}$ sheet. ${ }^{10}$

In addition to nanotubes, graphene is considered as a capable candidate for monitoring and controlling toxic gases. The defective or doped graphene shows stronger adsorption rather than the pristine structures. ${ }^{11-13}$ Using first-principles calculations, it has been shown that the $\mathrm{Al}$-doped graphene is more sensitive to the adsorption of $\mathrm{CO}$ than pristine graphene and $\mathrm{N}$-doped graphene. ${ }^{5}$ The structural and electronic properties of the graphene-molecule adsorption are strongly dependent on the configuration of the molecular adsorption. ${ }^{11}$ 
The investigation of the electronic structure of the SWNT's has been carried out by STS.

Investigation of the electronic structure of gas molecules adsorbed on GNRs by the tight-binding
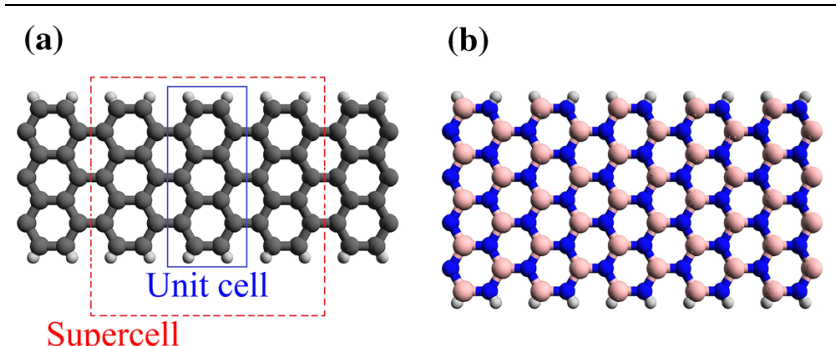

(c)

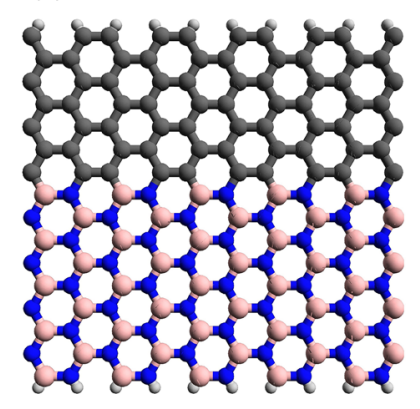

(d)

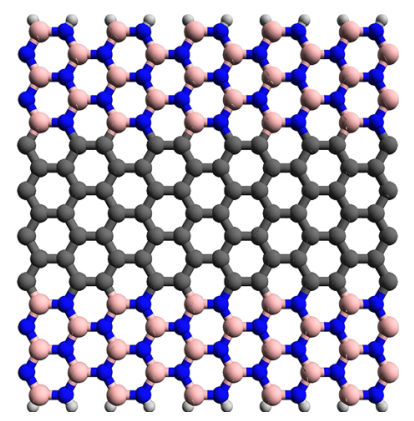

Fig. 1. The geometrical structure of (a) pristine $A_{7} G N R$, (b) pristine $A_{9} B N N R$, (c) pristine $A_{9} B N N R-A_{7} G N R$, and (d) pristine $A_{5} B N N R-$ $A_{7}$ GNR-A $A_{5} B N N R$ systems. A supercell which is three times larger than the unit cell is shown in panel (a).
DFT based calculation, has shown that the new states induced by $\mathrm{CO}$ and $\mathrm{NH}_{3}$ molecules are localized near the Fermi level and the conduction band, respectively. ${ }^{14}$ The electronic and transport properties of the AGNRs are sensitive to the gas adsorption and their conductance is more affected by $\mathrm{NH}_{3}$ rather than $\mathrm{CO}$ molecules. ${ }^{15}$

Using tight binding theory, it was found that the energy gap of doped carbon nanotubes is reduced by $\mathrm{B} / \mathrm{N}$-doping, and the reduction value is sensitive to the nanotube diameter and chirality, impurity type and concentration. ${ }^{16}$ In BNNTs, the band gap is reduced due to substitution of $\mathrm{B} / \mathrm{N}$ atoms by carbon atoms, and the doping effects of boron and nitrogensubstituted BNNTs are different. ${ }^{17}$

Another type of BCN nanostructure is BN-C hybrids of nanoribbons, which are composed by GNRs embedded in BNNRs. BN-C hybrid nanoribbons have a stable honeycomb configuration ${ }^{18}$ and can be synthesized by the CVD methods. ${ }^{19}$ The BCN nanostructures could be good alternatives for $\mathrm{CO}$ and $\mathrm{NH}_{3}$ detecting instead of pristine graphene allotropes or pure BN structures.

The electronic properties of these insulator or semiconductor structures can be changed to metals or half-metals by tuning the concentration of the carbon atoms. ${ }^{18-21}$ Their tunable electronic properties suggest potential applications of BNC hybrid nanoribbons in nano-electronic and nano-sensors for detecting low concentrations of molecules.

Table I. The reported tight-binding parameters for the BN-C hybrid nanoribbons ${ }^{20}$

\begin{tabular}{llllllllll}
\hline $\begin{array}{l}\boldsymbol{t}_{\mathbf{C}-\mathbf{C}} \\
0.839\end{array} \quad \frac{\boldsymbol{t}_{\mathbf{N}-\mathbf{N}}}{0.161}$ & $\frac{\boldsymbol{t}_{\mathbf{B}-\mathbf{C}}}{0.75}$ & $\frac{\boldsymbol{t}_{\mathbf{N}-\mathbf{C}}}{0.857}$ & $\frac{\boldsymbol{t}_{\mathbf{B}-\mathbf{N}}}{1}$ & $\frac{\boldsymbol{t}_{\mathbf{B}-\mathbf{C}, \mathbf{2}}}{0.036}$ & $\frac{\boldsymbol{t}_{\mathbf{N}-\mathbf{C}, \mathbf{2}}}{0.107}$ & $\frac{\varepsilon_{\mathbf{C}}}{0.0054}$ & $\frac{\varepsilon_{\mathbf{B}}}{0.732}$ & $\frac{\varepsilon_{\mathbf{N}}}{-0.732}$
\end{tabular}

All parameters are determined in terms of $t_{\mathrm{B}-\mathrm{N}}$.

Table II. The reported tight-binding parameters for the adsorption of the carbon monoxide and ammonia molecules on the GNRs ${ }^{13}$

\begin{tabular}{|c|c|c|c|c|c|c|c|}
\hline $\boldsymbol{t}_{\mathbf{C}-\mathbf{O}}$ & $t_{\mathrm{N}-\mathrm{H}}$ & $\boldsymbol{t}_{\mathrm{C}_{\mathrm{GNR}}-\mathrm{CO}}$ & $\boldsymbol{t}_{\mathbf{C}_{\mathbf{G N R}}-\mathbf{N H}_{3}}$ & $\varepsilon_{\mathbf{C}}$ & $\varepsilon_{\mathbf{O}}$ & $\varepsilon_{\mathbf{N}}$ & $\varepsilon_{\mathbf{H}}$ \\
\hline 1.379 & 1.804 & 1.054 & 0.854 & -1.329 & -1.329 & -3.279 & -2.518 \\
\hline
\end{tabular}

All parameters are determined in terms of $t_{\mathrm{B}-\mathrm{N}}$.

Table III. Our proposed tight-binding parameters for the $\mathrm{CO}$ and $\mathrm{NH}_{3}$ adsorption on the BNNRs using DFT results ${ }^{8,29,30}$

\begin{tabular}{|c|c|c|c|c|c|c|c|c|c|}
\hline$t_{\mathbf{C}-\mathbf{O}}$ & $t_{\mathrm{N}-\mathrm{H}}$ & $t_{\mathrm{B}_{\mathrm{BNR}}-\mathrm{CO}}$ & $t_{\mathrm{N}_{\mathrm{BNR}}-\mathrm{CO}}$ & $t_{\mathrm{B}_{\mathrm{BNR}}-\mathrm{NH}_{3}}$ & $t_{\mathbf{N}_{\mathrm{BNR}}-\mathbf{N H}_{3}}$ & $\varepsilon_{\mathbf{C}}$ & $\varepsilon_{\mathbf{O}}$ & $\varepsilon_{\mathbf{H}}$ & $\varepsilon_{\mathbf{N}}$ \\
\hline 1.525 & 1.9 & 0.243 & 0.282 & 0.175 & 0.136 & 2.143 & 2.143 & -0.75 & -1 \\
\hline
\end{tabular}

All parameters are determined in terms of $t_{\mathrm{B}-\mathrm{N}}$. 


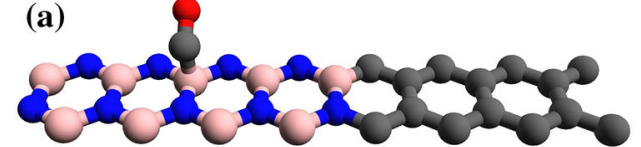

(c)

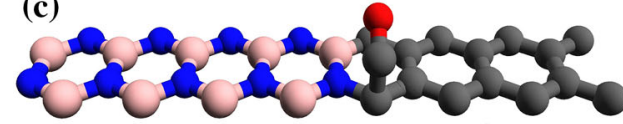

(e)

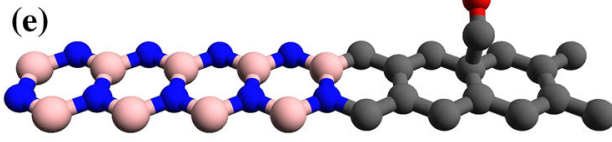

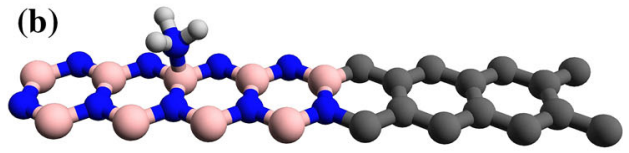

(d)

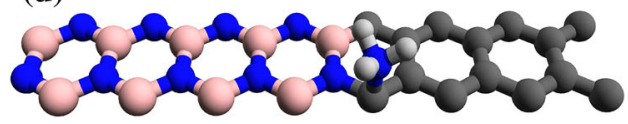

(f)

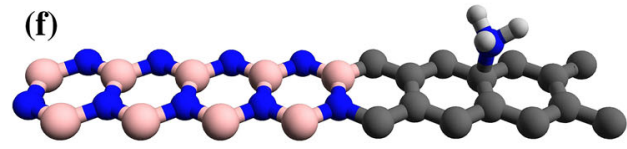

Fig. 2. Some geometrical molecule adsorption configurations of $\mathrm{A}_{9} B N N R-\mathrm{A}_{7} \mathrm{GNR}$. In part (a) and (b) the $\mathrm{CO}$ and $\mathrm{NH}_{3}$ molecules adsorbed to central boron atom, respectively. In part (c) and (d) the $\mathrm{CO}$ and $\mathrm{NH}_{3}$ adsorbed on carbon atoms at the boundary of the GNR and BNNR region. In part (e) and (f) the $\mathrm{CO}$ and $\mathrm{NH}_{3}$ adsorbed to the central carbon atom, respectively.

Due to random distribution of gas adsorption, the translational symmetry of a real disordered system is broken for these systems. Because of a large number of possible configurations, the physical quantities must be obtained via the average of all configurations. The disordered systems can be evaluated by averaging approaches such as coherent potential approximation $(\mathrm{CPA})^{22}$ or the transfer matrix method. ${ }^{14}$ In this study we have investigated the effects of $\mathrm{CO}$ and $\mathrm{NH}_{3}$ adsorption on $\mathrm{BN}-\mathrm{C}$ hybrid nanoribbons by using the NEGF technique.

The remainder of this paper is organized as follows. In the next section, we explain the theoretical aspects of our work. In the "Results and Discussion" section, we present the dependence of densities of states (DOS) spectrum, quantum conductance and current-voltage characteristics on gas (CO and $\mathrm{NH}_{3}$ ) adsorption. The conclusion of our work is represented in "Conclusions" section.

\section{MODEL AND FORMALISM}

The nanoribbon can be described as the linear supercell chains divided into a central supercell surrounded by two semi-infinite stacks. We assume that adsorption occurs on the center area and this area contains several unit cells including random distribution of gas molecules. For the center region by $N_{S}$ atoms and $N_{G}$ molecule adsorption, the gas concentration is determined as $c=\frac{N_{G}}{N_{s}} .14$ In this study we suppose $N_{G}=1$. The possibility weight of each configuration can be attributed to the adsorption energy $\left[E_{\text {ads }}=E_{\text {adsorbed }}-\left(E_{\text {pristine }}+E_{\text {molecule }}\right)\right]$ with the linear relation. The bond length and hopping integrals for nanoribbon edge atoms change due to hydrogen passivation. ${ }^{23}$

The on-site energy for the edges and middle atoms is differ due to their different chemical environments. ${ }^{23}$

The electronic properties of the system including semi-infinite regions can be investigated by using of surface Green's function and transfer matrix approach. ${ }^{24}$ For the nonmagnetic system, in the
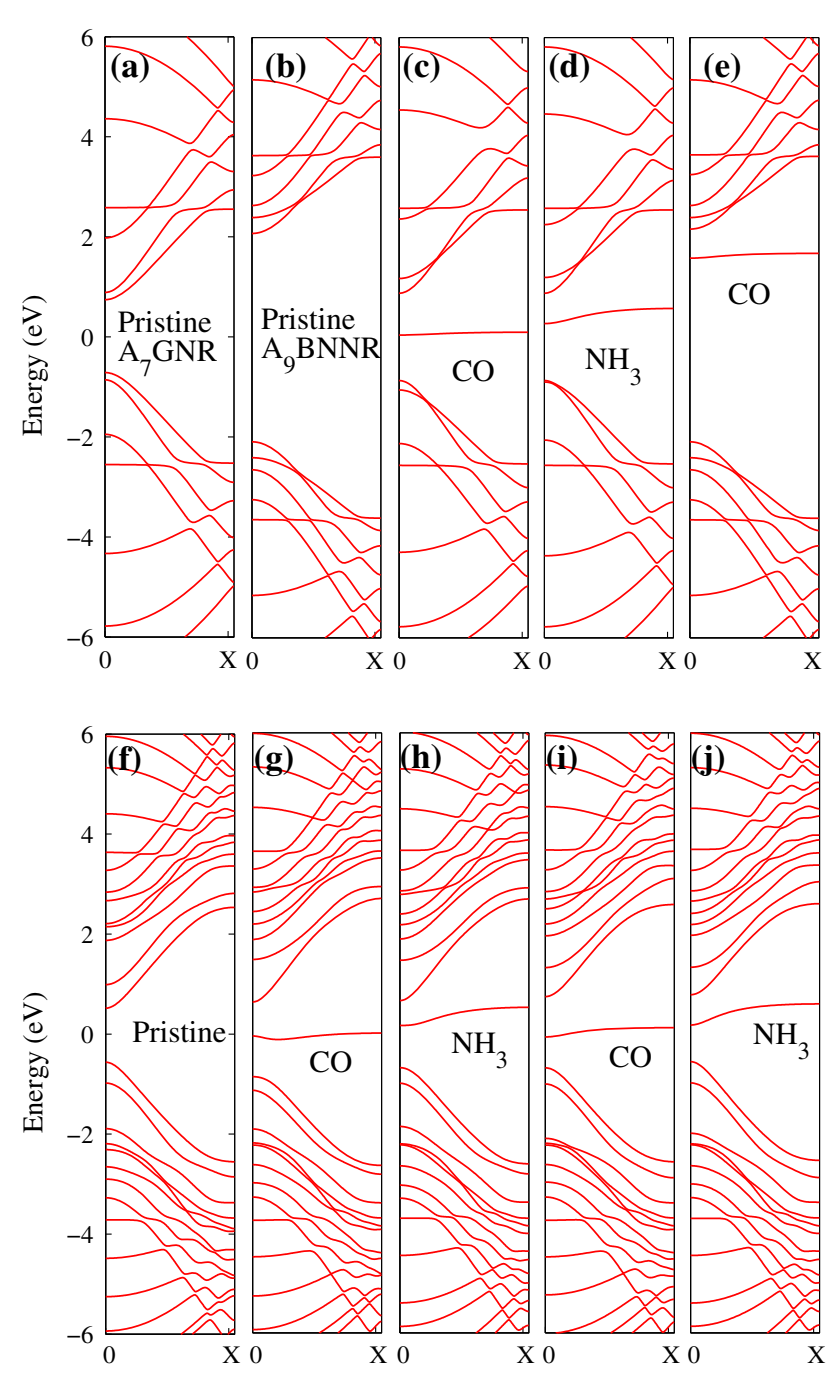

Fig. 3. Electronic band structure of (a) pristine $A_{7} G N R$, (b) pristine $A_{9} B N N R$, (c) $A_{7}$ GNR with CO adsorption, (d) $A_{7}$ GNR with $\mathrm{NH}_{3}$ adsorption, (e) $A_{9} B N N R$ with $C O$ adsorption, (f) pristine $A_{9} B N N R-$ $A_{7} G N R$, (g) $A_{9} B N N R-A_{7} G N R$ with $C O$ adsorption (corresponding to Fig. 2c), (h) $A_{9} B N N R-A_{7} G N R$ with $\mathrm{NH}_{3}$ adsorption (corresponding to Fig. 2d), (i) $A_{9} B N N R-A_{7} G N R$ with $C O$ adsorption (corresponding to Fig. $2 e$, (j) $A_{9} B N N R-A_{7} G N R$ with $\mathrm{NH}_{3}$ adsorption (corresponding to Fig. 2f). In the panels (c)-(e), the gas molecule adsorbed to the one of central carbon (boron) sites in $A_{7}$ GNR ( $\left.A_{9} B N N R\right) . X= \pm \pi / a$, and $a$ is the length of the unit cell. 


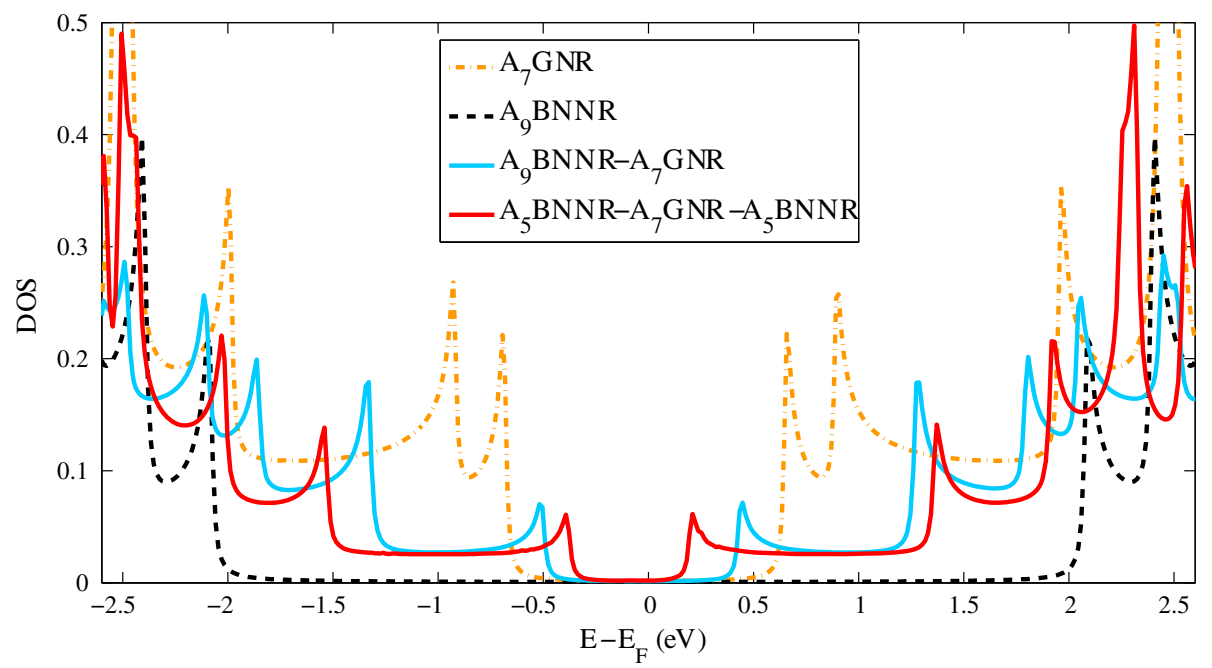

Fig. 4. Total DOS of pristine $A_{7} G N R$, pristine $A_{9} B N N R$, pristine $A_{9} B N N R-A_{7} G N R$ and pristine $A_{5} B N N R-A_{7} G N R-A_{5} B N N R$.
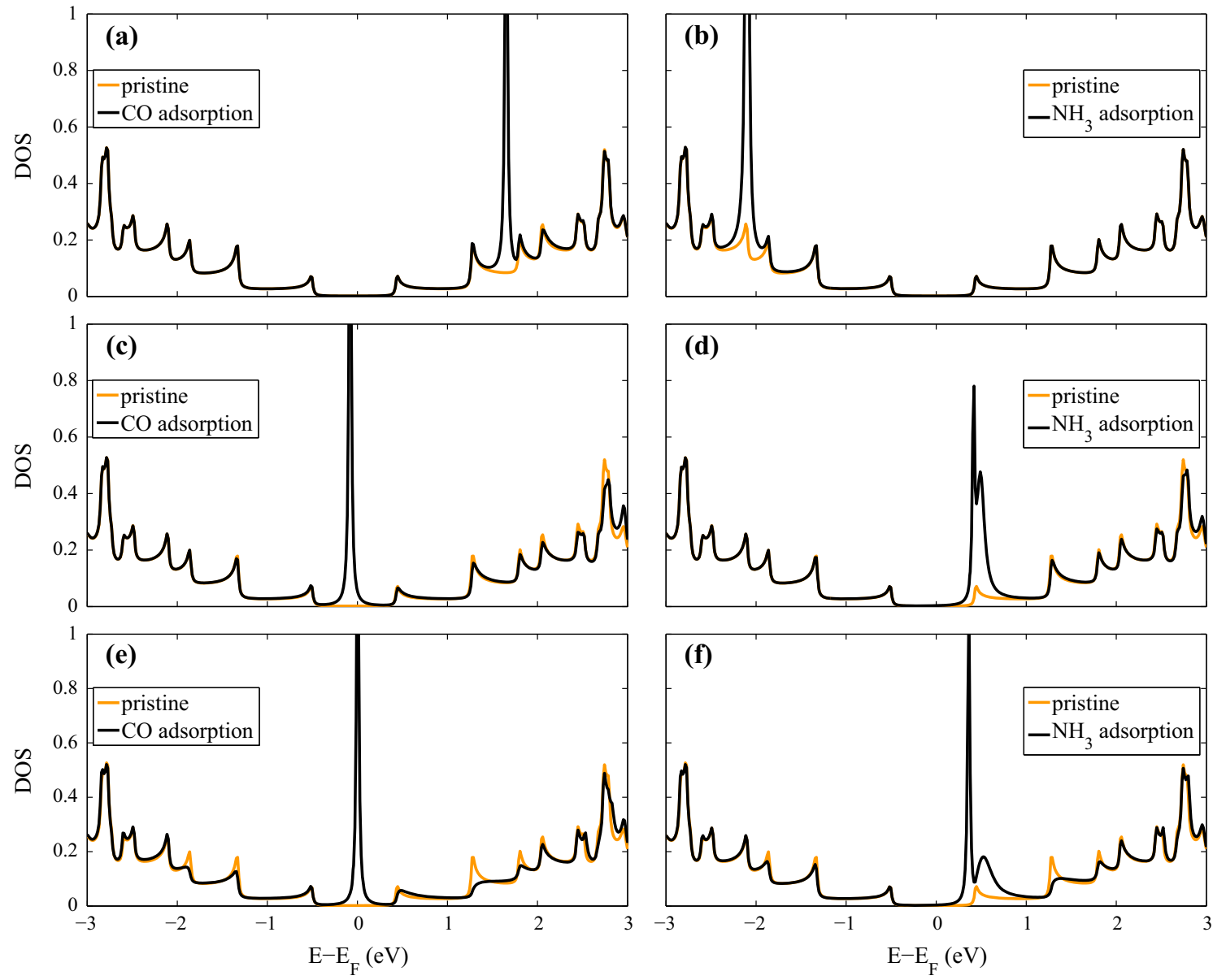

Fig. 5. DOS of some adsorption configurations of $A_{9} B N N R-A_{7} G N R$. The parts (a)-(f) show the DOS corresponding to adsorption configurations of the Fig. $2 a, b, c, d, e$, and f, respectively. 

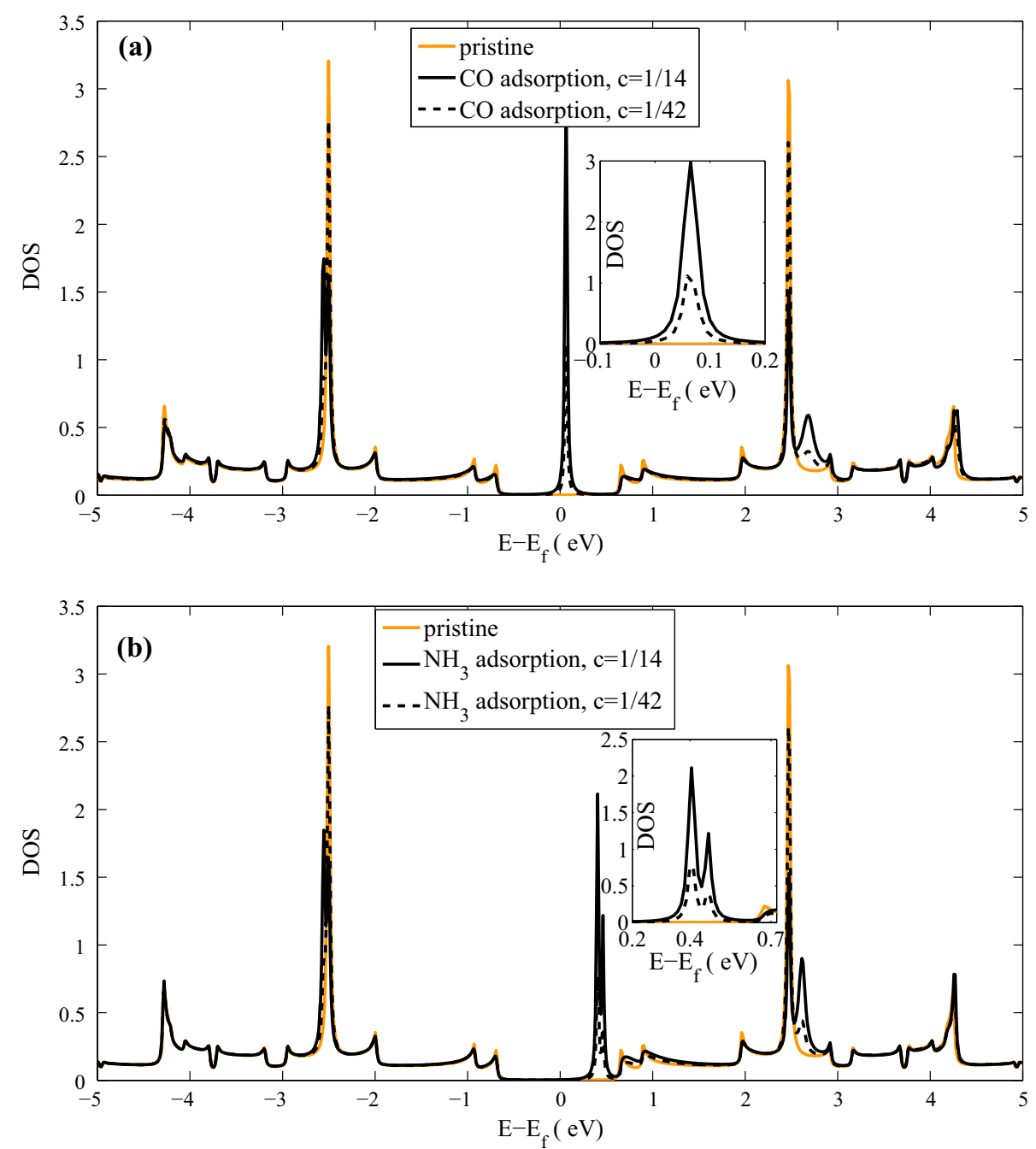

Fig. 6. Panel (a) and the inset, show the average DOS of $A_{7} G N R$ with $C O$ adsorption for two values of the gas concentration $c=\frac{1}{14}$ and $c=\frac{1}{42}$. The average DOS of $\mathrm{A}_{7} \mathrm{GNR}$ with $\mathrm{NH}_{3}$ adsorption for two values of the gas concentration $c=\frac{1}{14}$ and $c=\frac{1}{42}$, are illustrated in panel (b) and inset.

tight-binding approximation, the Hamiltonian of supercells is defined as ${ }^{14}$ :

$$
\begin{gathered}
H_{\alpha}=\sum_{\langle i \neq j\rangle} t_{i j} c_{i}^{\dagger} c_{j}+\sum_{i} \varepsilon_{i} \hat{n}_{i}, \quad \alpha=\ldots, \\
L-1, L, C, R, R+1, \ldots
\end{gathered}
$$

where $\alpha$ refer to left, right and central $(L, R$ and $C)$ supercells ( $\alpha=\ldots, L-1, L, C, R, R+1, \ldots$ ). The $t_{i j}$ is the hopping integral, $\varepsilon_{i}$ is the on-site energy, $c_{i}^{\dagger}\left(c_{i}\right)$ is the create (annihilate) operator at site $i$. In the presence of an adsorption molecule, the Hamiltonian of the central supercell is obtained by:

$$
H_{C}^{i}=H_{C}+H_{\mathrm{imp}}^{i},
$$

where $H_{\text {imp }}^{i}$ is the Hamiltonian for the adsorbed molecule at $i$ th site and determined by ${ }^{14}$ :

$$
\begin{aligned}
H_{\mathrm{imp}}^{i}= & \sum_{\ell_{i}} \varepsilon_{\ell_{i}} d_{\ell_{i}}^{\dagger} d_{\ell_{i}}+\sum_{\ell_{i} \ell_{i}^{\prime}} t_{\ell_{i} \ell_{i}^{\prime}} d_{\ell_{i}}^{\dagger} d_{\ell_{i}^{\prime}} \\
& +\sum_{i} \sum_{\ell_{i}} t_{i \ell_{i}}\left(c_{i}^{\dagger} d_{\ell_{i}}+d_{\ell_{i}}^{\dagger} c_{i}\right),
\end{aligned}
$$

where $d_{\ell_{i}}\left(d_{\ell_{i}}^{\dagger}\right)$ is the create (annihilate) operator in the orbital $\ell$ of the molecule located at the $i$ th ribbon site, the $\varepsilon_{\ell_{i}}$ and $t_{\ell_{i} \ell_{i}^{\prime}}$ are the on-site energy and hopping parameters for orbitals in the molecule, respectively. The $t_{i \ell_{i}}$ is the hopping parameter between the molecule and ribbon. The total Green's function for any adsorbed configuration can be calculated by ${ }^{25}$ :

$$
G^{i}(E)=\left(E^{+}-H_{C}^{i}-\Sigma_{L}-\Sigma_{R}\right)^{-1}
$$

where $E^{+}=E+i 0^{+}$and, $\Sigma_{L}\left(\Sigma_{R}\right)$ is the self-energy due to the interaction between the supercell and left 

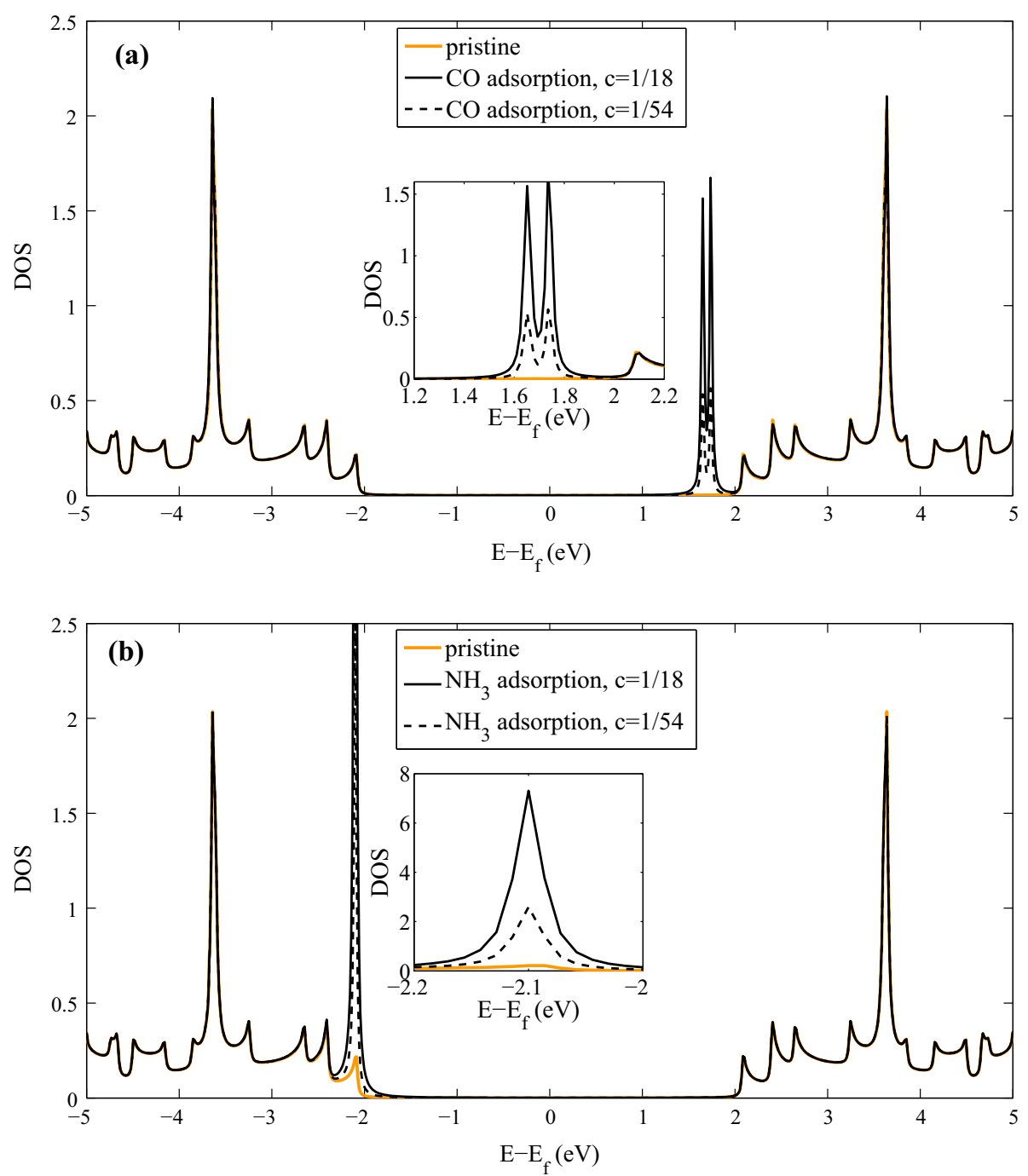

Fig. 7. Panel (a) and the inset, show the average DOS of $A_{9} B N N R$ with $C O$ adsorption for two values of the gas concentration $c=\frac{1}{18}$ and $c=\frac{1}{54}$. Panel (b) and inset, show the average DOS of $\mathrm{A}_{9} \mathrm{BNNR}$ with $\mathrm{NH}_{3}$ adsorption for two values of the gas concentration $c=\frac{1}{18}$ and $c=\frac{1}{54}$.

(right), which is estimated by an iteration method. For an $X$-type molecule, the average DOS overall configurations evaluated as $^{14}$ :

$$
\langle D(E)\rangle=-\frac{1}{\pi} \sum_{i} w_{i}^{X} \operatorname{Tr}\left(\operatorname{Im} G^{i}(E)\right) .
$$

Here, $w_{i}^{X}$ is the probability weight for the $i$ th configuration. The transmission coefficient and quantum conductance, $Q_{C}(E)$ of the system can be determined $\mathrm{as}^{26,27}$ :

$$
\begin{aligned}
& T(E)=\operatorname{Tr}\left[\Gamma^{L} G(E) \Gamma^{R} G^{\dagger}(E)\right], \\
& \Gamma^{L, R}=i\left[\Sigma^{L, R}-\left(\Sigma^{L, R}\right)^{\dagger}\right] \\
& Q_{c}(E)=\frac{2 e^{2}}{h} T(E)
\end{aligned}
$$

where the $\Gamma^{L, R}$ is the coupling matrix on the left or right electrodes. The total current across the system $(I(V))$ is calculated $\mathrm{as}^{27}$ :

$$
I(V)=(2 e / h) \int_{-\infty}^{+\infty} T(E)\left[f\left(E-\mu_{L}\right)-f\left(E-\mu_{R}\right)\right] \mathrm{d} E,
$$

where $f(E)$ is the Fermi-Dirac distribution function.

\section{RESULTS AND DISCUSSION}

Figure 1 shows the schematic views of pristine $\mathrm{A}_{7} \mathrm{GNR}, \mathrm{A}_{9} \mathrm{BNNR}, \mathrm{A}_{9} \mathrm{BNNR}-\mathrm{A}_{7} \mathrm{GNR}$ and pristine $\mathrm{A}_{5} \mathrm{BNNR}-\mathrm{A}_{7} \mathrm{GNR}-\mathrm{A}_{5} \mathrm{BNNR}$ systems. Several parameters such as on-site energies of carbon, boron and nitrogen, hydrogen and oxygen atoms and the hopping integral between them are required to calculate the electronic properties of these systems. The hopping parameters and on-site energies 

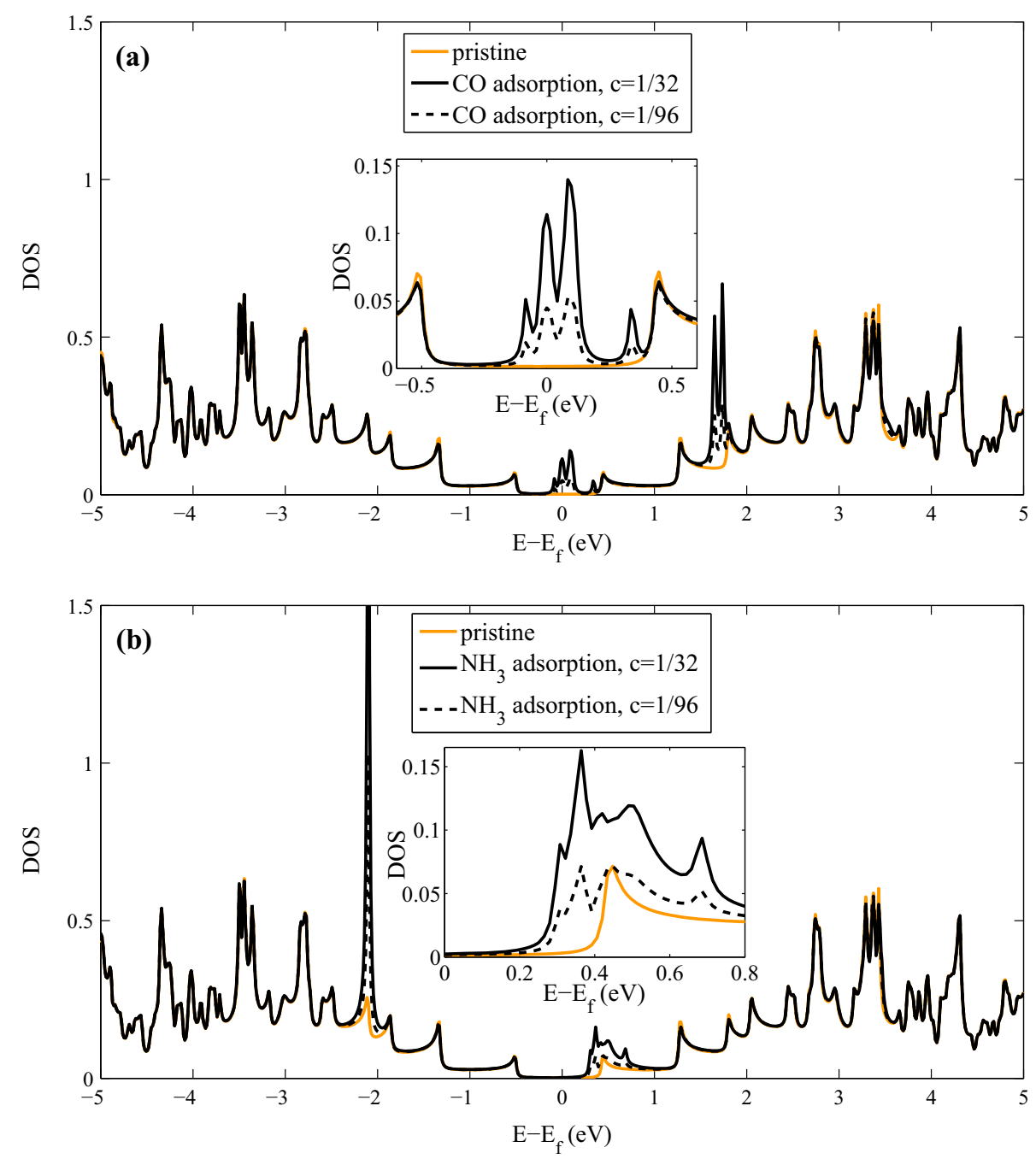

Fig. 8. Panel (a) and the inset, show the average DOS of $A_{9} B N N R-A_{7} G N R$ with $C O$ adsorption for two values of the gas concentration $c=\frac{1}{32}$ and $c=\frac{1}{96}$. Panel (b) and inset, show the average DOS of $\mathrm{A}_{9} \mathrm{BNNR}-\mathrm{A}_{7} \mathrm{GNR}$ with $\mathrm{NH}_{3}$ adsorption for two values of the gas concentration $c=\frac{1}{32}$ and $c=\frac{1}{96}$

of pristine BN-C hybrid nanoribbons and GNRs with molecules adsorption are shown in Tables I and II. ${ }^{14,20}$ The hopping parameters for BNNRs with $\mathrm{CO}$ and $\mathrm{NH}_{3}$ adsorption can be obtained by ${ }^{28}$ :

$$
t_{\alpha \beta}=t\left(\frac{d}{d_{\alpha \beta}}\right)^{2},
$$

where $d=1.42 \AA, d_{\alpha \beta}$ is the bond length between molecule and ribbon atoms and $t$ is the hopping parameter. Note that for all systems, the $d_{\alpha \beta}$ is determined from previous DFT calculations (Table III). The on-site energies can be obtained with varying $\varepsilon_{\ell_{i}}$ to achieve the results in agreement with the reported DFT results. ${ }^{4,29-31}$

Based on DFT calculations, the adsorption energy of $\mathrm{CO}$ on the GNRs and BNNRs is approximately equal $(0.13 \mathrm{eV})$ for $\mathrm{B} / \mathrm{N} / \mathrm{C}$ atoms. ${ }^{4,31,32}$ Based on the equivalence of the adsorption energy for each combination of the GNRs and BNNRs, ${ }^{20,33}$ we used the same probability weight for CO-adsorption on each of the B/N/C sites (similar to Ref. 14). Due to the DFT calculations, the adsorption energy of the adsorbed $\mathrm{NH}_{3}$ molecule on $\mathrm{B} / \mathrm{N} / \mathrm{C}$ atoms is equal to $0.112 \mathrm{eV}, 0.4 \mathrm{eV}$, and $0.48 \mathrm{eV}$, respectively. ${ }^{29,34}$ For molecules with neglected interactions (far from the ribbon's surface), the adsorption probability weight depends on the ribbon's area. For the $\mathrm{NH}_{3}$ molecules sufficiently close to the ribbon area, the adsorption probability weight is different for $\mathrm{B} / \mathrm{N} /$ $\mathrm{C}$ atoms. We defines the probability weight for $\mathrm{NH}_{3}$ adsorption on $\mathrm{B} / \mathrm{N} / \mathrm{C}$ atoms as:

$$
\begin{aligned}
w_{\mathrm{C}}^{\mathrm{NH}_{3}} & =\frac{S_{\mathrm{GNR}}}{N_{\mathrm{GNR}} \times S_{\mathrm{T}}} \\
w_{\mathrm{B}}^{\mathrm{NH}_{3}} & =\frac{S_{\mathrm{BNNR}}}{N_{\mathrm{BNNR}} \times S_{\mathrm{T}}} \times \frac{E_{\mathrm{B}}^{\mathrm{ads}}}{E_{\mathrm{B}}^{\mathrm{ads}}+E_{\mathrm{N}}^{\mathrm{ads}}}, \\
w_{\mathrm{N}}^{\mathrm{NH}_{3}} & =\frac{S_{\mathrm{BNNR}}}{N_{\mathrm{BNNR}} \times S_{\mathrm{T}}} \times \frac{E_{\mathrm{N}}^{\mathrm{ads}}}{E_{\mathrm{B}}^{\mathrm{ads}}+E_{\mathrm{N}}^{\mathrm{ads}}}
\end{aligned}
$$



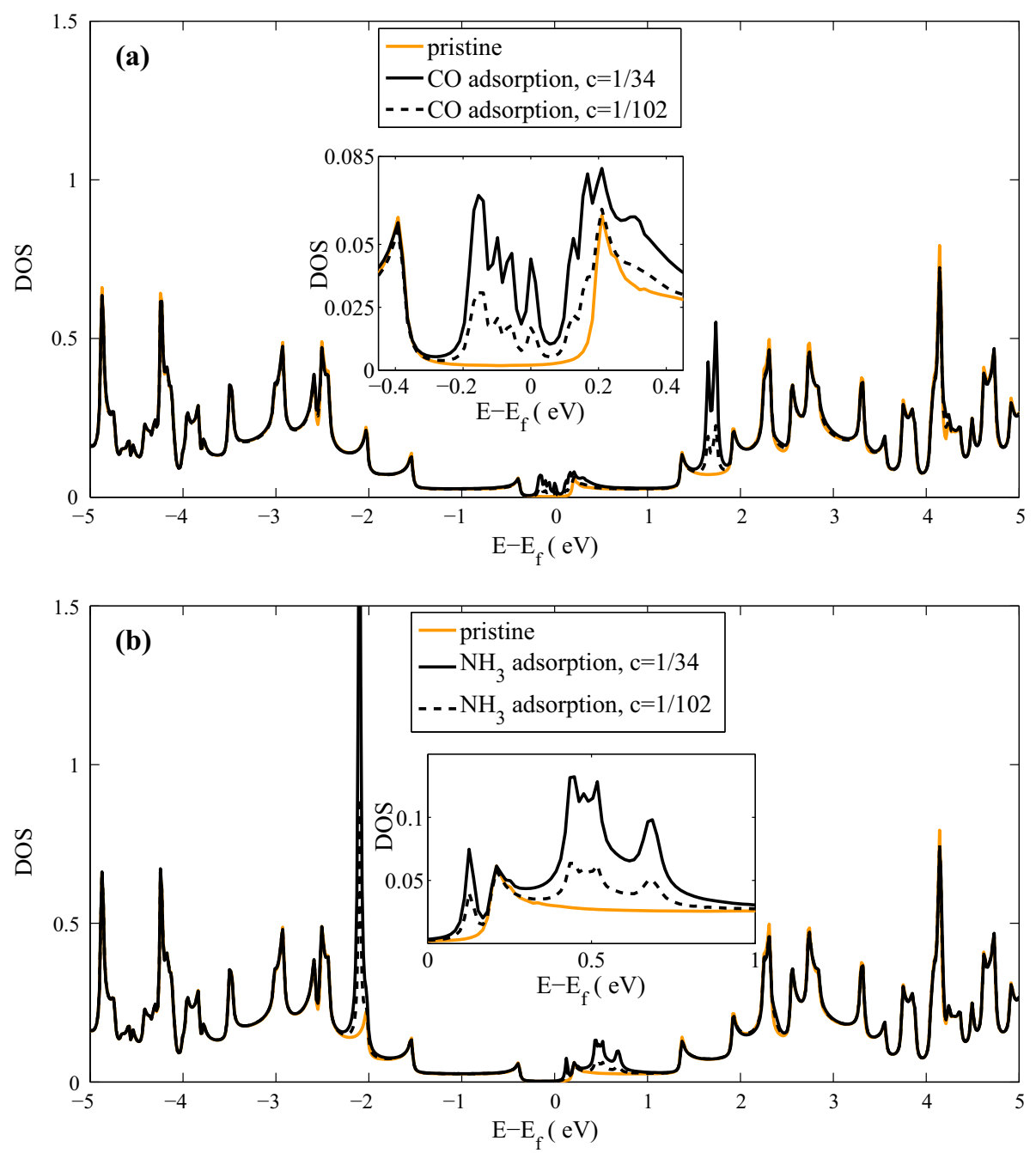

Fig. 9. Panel (a) and the inset, show the average DOS of $A_{5} B N N R-A_{7} G N R-A_{5} B N N R$ with $C O$ adsorption for two values of the gas concentration $c=\frac{1}{34}$ and $c=\frac{1}{102}$. Panel (b) and inset, show the average DOS of $A_{5} B N N R-A_{7} G N R-A_{5} B N N R$ with $N_{3} H_{3}$ adsorption for two values of the gas concentration $c=\frac{1}{34}$ and $c=\frac{1}{102}$.

where $S_{\mathrm{GNR}}\left(S_{\mathrm{BNNR}}\right)$ is surface area of GNR (BNNR) regions, respectively. $S_{\mathrm{T}}$ is total surface area of the supercell. The $N_{\mathrm{GNR}}\left(N_{\mathrm{BNNR}}\right)$ are the total number of sites of GNR (BNNR) in the supercell. $E_{\mathrm{B}}^{\mathrm{ads}}\left(E_{\mathrm{N}}^{\mathrm{ads}}\right)$ is the adsorption energy of $\mathrm{NH}_{3}$ on the boron (nitrogen) atom.

\section{Electronic Structure}

Figure 2 shows some geometrical molecule adsorption configurations of $\mathrm{A}_{9} \mathrm{BNNR}-\mathrm{A}_{7} \mathrm{GNR}$. The calculated band structure of $\mathrm{A}_{7} \mathrm{GNR}$ and $\mathrm{A}_{9} \mathrm{BNNR}$ with different $\mathrm{CO}$ and $\mathrm{NH}_{3}$ gas adsorptions are shown in Fig. 3c, d, and e. For a periodic configuration in the $\mathrm{A}_{7} \mathrm{GNR}$ ( $\mathrm{A}_{9} \mathrm{BNNR}$ ), the gas molecule is adsorbed on the carbon (boron) atoms at the center of the nanoribbon. After $\mathrm{CO}$ and $\mathrm{NH}_{3}$ adsorption in the $A_{7} G N R$, the impurity level is created above Fermi level in the conduction bands (in agreement with Ref. 14) and the system becomes a $n$-type semiconductor (Fig. $3 c$ and d). In the $\mathrm{A}_{9} \mathrm{BNNR}$, the $\mathrm{CO}$ adsorption creates a new band above the Fermi level (below the lowest conduction bands), narrows the band gap and slightly shifts the Fermi level toward the conduction band (Fig. 3e). The charge transfer is positive for these donor cases in agreement with Ref. 35. Figure $3 g, h, i$, and $\mathrm{j}$ shows the calculated the band structure of $\mathrm{A}_{7}$ GNR-A $\mathrm{A}_{9} \mathrm{BNNR}$ hybrid system with different $\mathrm{CO}$ and $\mathrm{NH}_{3}$ gas adsorption (corresponding to Fig. 2c, d, e, and f). For all selected cases, the gas adsorption creates a new band above the Fermi level.

\section{Density of States}

In the Fig. 4 we compare the total DOS of pristine $\mathrm{A}_{7}$ GNR, $\mathrm{A}_{9} \mathrm{BNNR}, \mathrm{A}_{9} \mathrm{BNNR}-\mathrm{A}_{7} \mathrm{GNR}$ and $\mathrm{A}_{5} \mathrm{BNNR}-$ $\mathrm{A}_{7}$ GNR-A $\mathrm{A}_{5} \mathrm{BNNR}$ (corresponding to unit cells in 

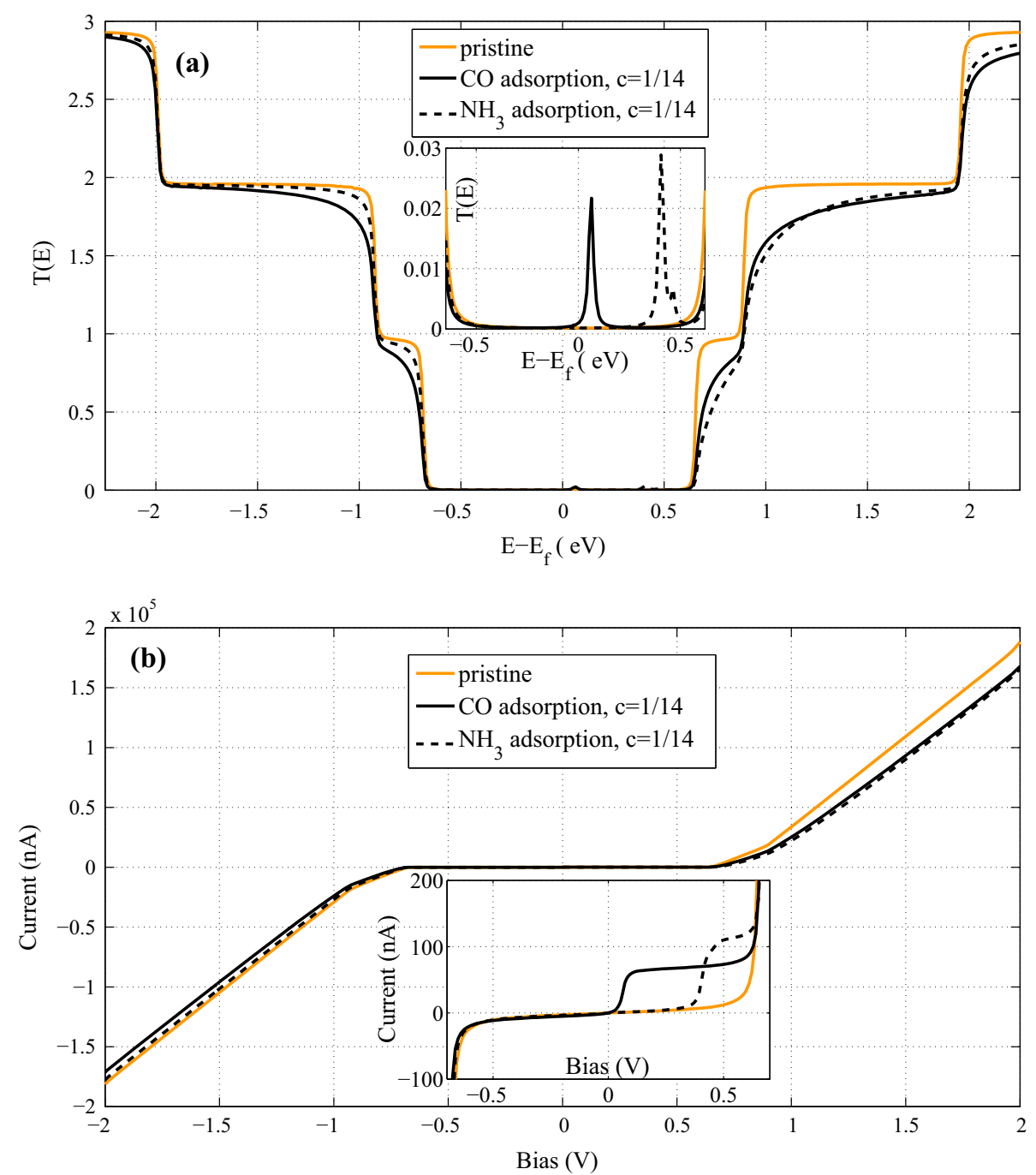

Fig. 10. Panel (a) and inset (panel (b) and inset), show the transmission coefficient ( $I-V$ characteristics) of $A_{7} G N R$ at concentration of $c=\frac{1}{14}$ for $\mathrm{CO}$ and $\mathrm{NH}_{3}$ adsorption.

Fig. 1). The gap is decreased to $0.94 \mathrm{eV}$ for (A9BNR-A ${ }_{7}$ GNR) hybrid system in comparison with the pristine $\mathrm{A}_{9} \mathrm{BNNR}\left(E_{\mathrm{g}}=4.2 \mathrm{eV}\right)$ and $\mathrm{A}_{7} \mathrm{GNR}\left(E_{\mathrm{g}}=1.35 \mathrm{eV}\right)$. Also, the gap is decreased to $0.58 \mathrm{eV}$ for the $\left(\mathrm{A}_{5} \mathrm{BN}-\mathrm{A}_{7} \mathrm{GNR}-\mathrm{A}_{5} \mathrm{BNNR}\right)$ hybrid system.

Figure 5a, b, c, d, e, and $\mathrm{f}$ shows DOS for the $\mathrm{A}_{9} \mathrm{BNNR}-\mathrm{A}_{7} \mathrm{GNR}$ hybrid system with various molecule adsorption positions (corresponding to Fig. 2a, $\mathrm{b}, \mathrm{c}, \mathrm{d}, \mathrm{e}$, and $\mathrm{f}$ ). By the $\mathrm{CO}$ and $\mathrm{NH}_{3}$ gas adsorption on the boron atoms (Fig. 2a and b), some modifications occur far from the Fermi level in the conduction and valence regions. After $\mathrm{CO}$ gas adsorption on the carbon atoms, a distinct sharp peak occurs around the Fermi level due to the band structure modifications (Fig. 2c and e). The $\mathrm{NH}_{3}$ gas adsorption on the carbon atoms leads to create dual peaks near the Fermi level at the conduction region due to the modifications of the band structure such as band splitting and creating a donor band (Fig. $2 \mathrm{~d}$ and f).
The effects of various concentrations of $\mathrm{CO}$ and $\mathrm{NH}_{3}$ adsorption molecules on the average DOS for $\mathrm{A}_{7} \mathrm{GNR}, \mathrm{A}_{9} \mathrm{BNNR}, \mathrm{A}_{9} \mathrm{BNNR}-\mathrm{A}_{7} \mathrm{GNR}$ and $\mathrm{A}_{5} \mathrm{BNNR}-$ $\mathrm{A}_{7}$ GNR-A ${ }_{5}$ BNNR systems are shown in Figs. 6, 7, 8, and 9, respectively. As shown in Fig. 6a (Fig. 6b) for $\mathrm{A}_{7} \mathrm{GNR}$, the $\mathrm{CO}\left(\mathrm{NH}_{3}\right)$ adsorbed molecule induces quasi-localized state in the band gap, which is located near and above the Fermi level (near the conduction band) without any changing in the position of the HOMO and LUMO states in good agreement with previous study. ${ }^{14}$ The decreasing of the concentration of gas leads to decreasing the intensity of the induced states (see Fig. 6). For this case, the modifications of DOS in positive energies are more significant than negative energies.

As shown in Fig. 7, for the $\mathrm{A}_{9} \mathrm{BNNR}$, the adsorbed CO molecule induces states near the LUMO state without changing in the position of HOMO state in agreement with previous studies. ${ }^{4,30,31}$ Increasing the gas concentration increases the intensity of 

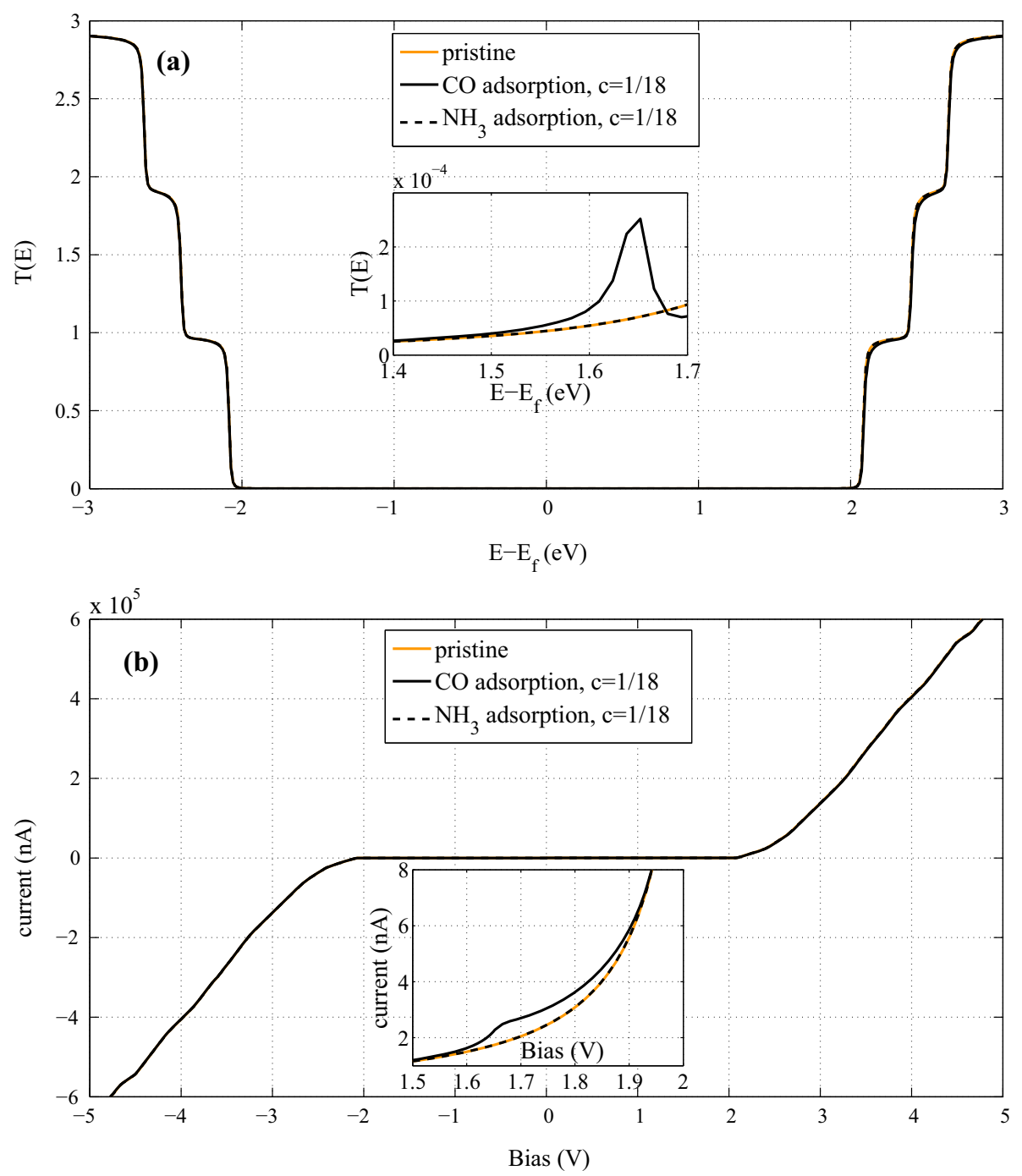

Fig. 11. Panel (a) and inset (panel (b) and inset), show the transmission coefficient ( $I-V$ characteristics) of $A_{9} B N N R$ at concentration of $c=\frac{1}{18}$ for $\mathrm{CO}$ and $\mathrm{NH}_{3}$ adsorption.

induced DOS peaks. In the presence of the $\mathrm{CO}$ molecule, the $\mathrm{A}_{9} \mathrm{BNNR}$ becomes like the $n$-type semiconductors. The intensity of HOMO state increases by $\mathrm{NH}_{3}$ adsorption. ${ }^{29}$ In the $\mathrm{A}_{9} \mathrm{BNNR}$, for both cases with different concentration, the modifications of electronic properties in higher and lower energies are neglected.

Figure 8 shows DOS of $\mathrm{A}_{9} \mathrm{BNNR} \mathrm{A}_{7} \mathrm{GNR}$ system in the presence of gas adsorption. Adsorption of the $\mathrm{CO}$ molecule to this system leads to modifications near the conduction band and creates several neighbor peaks in the band gap region with broadening energies about $0.2 \mathrm{eV}$ (Fig. 8a). For the $\mathrm{A}_{9} \mathrm{BNNR}-\mathrm{A}_{7} \mathrm{GNR}$ system, the $\mathrm{NH}_{3}$ gas induces new states and decreases the band gap by shifting the LUMO to the lower energies region. The system in the presence of $\mathrm{NH}_{3}$ gas behaves as the $n$-type semiconductor. The decreasing of gas concentration leads to decreasing the intensity of the induced states as shown in Fig. 8. The effects of $\mathrm{CO}$ and $\mathrm{NH}_{3}$ gas adsorption on average DOS of $\mathrm{A}_{5} \mathrm{BNNR}-\mathrm{A}_{7} \mathrm{GNR}-\mathrm{A}_{5} \mathrm{BNNR}$ are shown in Fig. 9. The domain of impurity states, which are induced by the $\mathrm{CO}$ molecule, contains energies below the Fermi level (Fig. 9a). The $\mathrm{NH}_{3}$ molecule creates impurity states near the conduction band and decreases the energy band gap. Also, the intensity of induced states is reduced by decreasing the gas concentration and the system becomes like $n$-type semiconductors (Fig. 9b).

\section{Conductance}

Figures 10, 11, 12, and 13 show the average transmission coefficient and current-voltage characteristics for $\mathrm{A}_{7} \mathrm{GNR}, \mathrm{A}_{9} \mathrm{BNNR}, \mathrm{A}_{9} \mathrm{BNNR}-\mathrm{A}_{7} \mathrm{GNR}$ and $\mathrm{A}_{5} \mathrm{BNNR}-\mathrm{A}_{7} \mathrm{GNR}-\mathrm{A}_{5} \mathrm{BNNR}$ systems in the presence of $\mathrm{CO}$ and $\mathrm{NH}_{3}$ gas adsorption, respectively. 

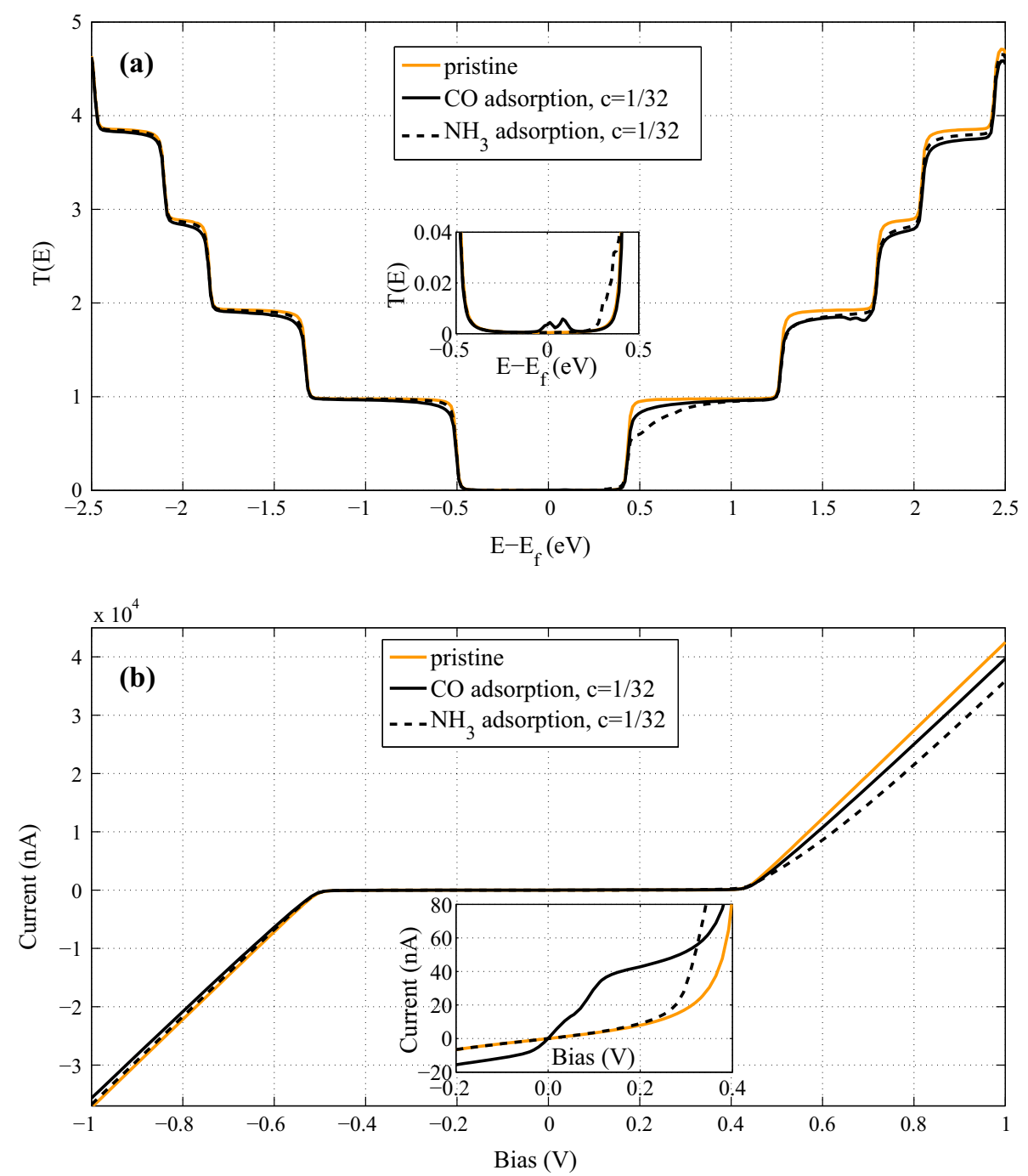

Fig. 12. Panel (a) and inset (panel (b) and inset), show the transmission coefficient ( $I-V$ characteristics) of $A_{9} B N N R-A_{7} G N R$ at concentration of $c=\frac{1}{32}$ for $\mathrm{CO}$ and $\mathrm{NH}_{3}$ adsorption.

These nanoribbons can be considered as a junction electrode in the $I-V$ characteristics calculations. As shown in these figures, the conductance is reduceds in energies far from Fermi level due to the gas adsorption.

For $\mathrm{A}_{7} \mathrm{GNR}$ in comparison with the pristine case, $\mathrm{CO}$ and $\mathrm{NH}_{3}$ molecules adsorption reduce the conductance and open the new conductance channels (Fig. 10a). These effects originate from electronic modifications after gas adsorption. For $\mathrm{A}_{7}$ GNR with $\mathrm{CO}$ and $\mathrm{NH}_{3}$ adsorption, the I-V characteristics exhibit staircase features in the bias voltage ranges $[+0.05 \mathrm{~V}$ to $+0.6 \mathrm{~V}]$ and $[+0.4 \mathrm{~V}$ to $+0.6 \mathrm{~V}]$, respectively (Fig. 10b). For positive and negative bias, each system acts as a switch (shown in Fig. 10b). For $\mathrm{CO}\left(\mathrm{NH}_{3}\right)$ adsorption, the turn-on voltage is $+0.05 \mathrm{~V}(+0.4 \mathrm{~V})$ and the $\mathrm{A}_{7} \mathrm{GNR}$ becomes like the $n$-type semiconductor in the presence of $\mathrm{CO}$ and $\mathrm{NH}_{3}$ gases.
In the $\mathrm{A}_{9} \mathrm{BNNR}$ system, due to the induced states near the Fermi energy, the difference between conductance of clean and impure systems is negligible (Fig. 11). Then, the pristine $\mathrm{A}_{9} \mathrm{BNNR}$ is not an appropriate choice for detecting $\mathrm{CO}$ and $\mathrm{NH}_{3}$ molecules compared with hybrid systems.

Due to conductance channels, which are located at the Fermi level (Fig. 12a), the current-voltage characteristic shows an Ohm like (linear) behavior around zero bias. The $\mathrm{A}_{9} \mathrm{BNNR}-\mathrm{A}_{7} \mathrm{GNR}$ acts like a metallic system due to $\mathrm{CO}$ molecule absorpbtion. The $\mathrm{NH}_{3}$ molecule creates new transmission channels above the Fermi level. The turn-on voltages of $\mathrm{A}_{9}$ BNNR-A $\mathrm{A}_{7} \mathrm{GNR}$ with $\mathrm{NH}_{3}$ adsorption is $+0.3 \mathrm{~V}$ in the forward bias (Fig. 12b). The transmission coefficient and I-V characteristics of the $\mathrm{A}_{5} \mathrm{BNNR}$ $\mathrm{A}_{7}$ GNR-A $\mathrm{A}_{5} \mathrm{BNNR}$ in the presence of gas are shown in Fig. 13. Due to anti-resonance effects of quasilocalized states, ${ }^{25}$ some dip can be observed in the 

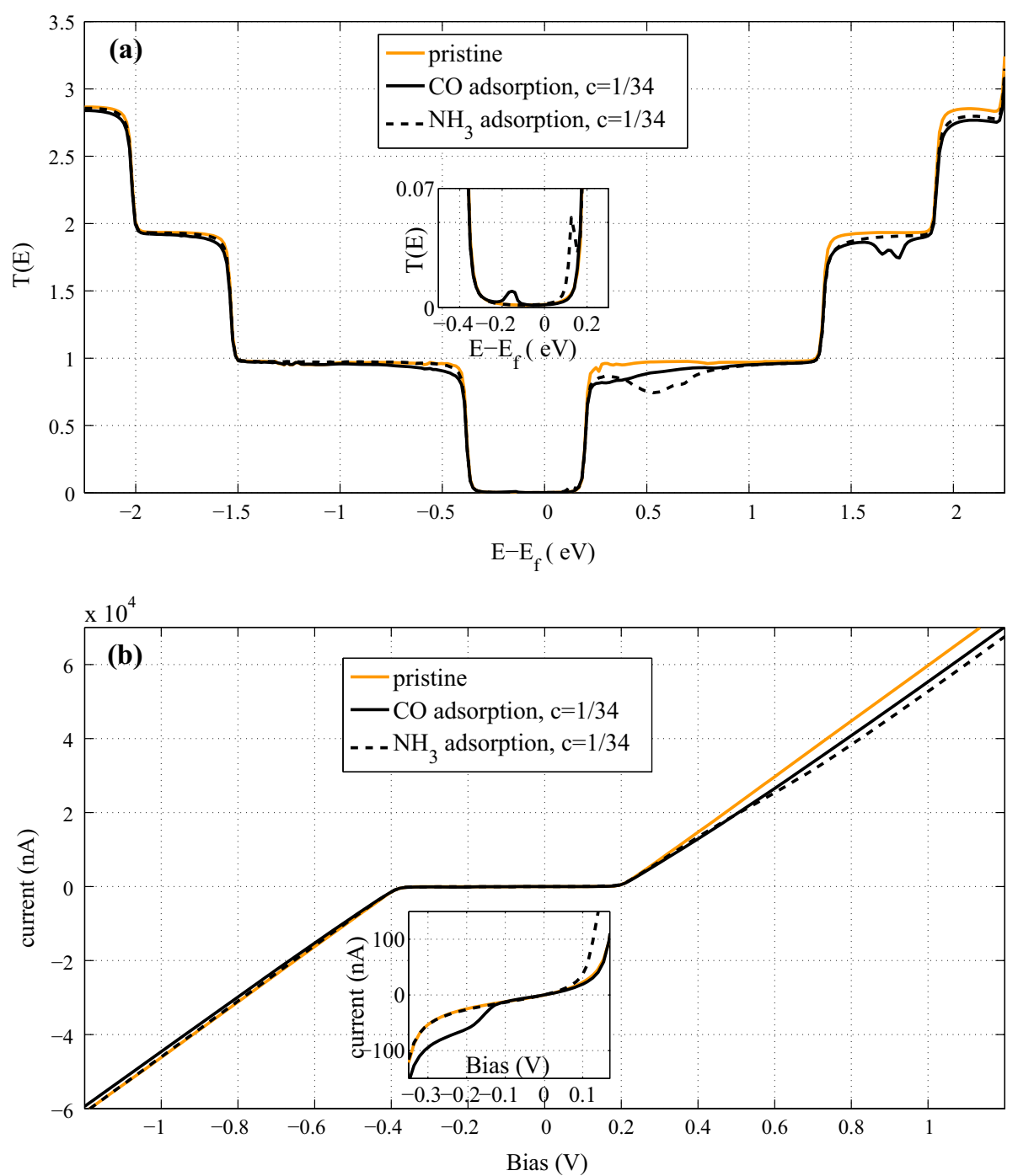

Fig. 13. Panel (a) and inset (panel (b) and inset), show the transmission coefficient ( $I-V$ characteristics) of $A_{5} B N N R-A_{7} G N R-A_{5} B N N R$ at concentration of $c=\frac{1}{34}$ for $\mathrm{CO}$ and $\mathrm{NH}_{3}$ adsorption.

transmission curves, while the conductance channels on the Fermi level have disappeared. This system in the presence of $\mathrm{CO}\left(\mathrm{NH}_{3}\right)$ molecule behaves as a $p$-type ( $n$-type) semiconductor.

\section{CONCLUSIONS}

In this research, the possibility of using AGNRs, BNNRs, and their hybrid compositions as the gas sensor is studied. For this purpose, we assume that only a single impurity molecule can be located in the supercell sites. The effects of adsorption on the electronic structure, average of the DOS, transmission coefficient and I-V characteristics overall random configurations are investigated by using NEGF technique. The required parameters have been obtained from previous DFT studies. The results indicate that, in BNNR, the randomly CO molecule adsorption induces states near the conductance band. The adsorption $\mathrm{NH}_{3}$ molecules increase intensity of the HOMO states. These adsorptions have negligible effects on conductance and $I-V$ characteristics for BNNRs compared with GNRs and hybrid systems. By embedding GNRs into BNNRs in the presence of $\mathrm{CO}$ and $\mathrm{NH}_{3}$ gas adsorption, the various and applicable electronic and conductance properties can be observed. For instance, the $\mathrm{A}_{9} \mathrm{BNNR}-\mathrm{A}_{7} \mathrm{GNR}\left(\mathrm{A}_{5} \mathrm{BNNR}-\mathrm{A}_{7} \mathrm{GNR}-\mathrm{A}_{5} \mathrm{BNNR}\right)$ system behaves as a metallic ( $p$-type semiconductor) system in the presence of $\mathrm{CO}$ adsorption. The gap of hybrid systems are decreased in the presence of $\mathrm{NH}_{3}$ gas, and they act like the $n$-type semiconductors. Our results show that these hybrid structures, due to their tunable band gap, can be better candidates for $\mathrm{CO}$ and $\mathrm{NH} 3$ gas detectors as compared to the pristine BNNRs and GNRs. Due to the various interesting electronic properties, the AGNR's embedded in BNNRs (BN-C hybrid 
nanoribbons) can be appropriate structures for the future of nanoelectronic devices. Especially, this hybrid structure can be a good candidate for CO and $\mathrm{NH}_{3}$ gas detecting as compared to the pristine BNNRs.

\section{REFERENCES}

1. Y.K. Yap, BCN nanotubes and related nanostructures (New York: Science, 2009).

2. J. Kong, N.R. Franklin, C. Zhou, M.G. Chapline, S. Peng, K. Cho, and H. Dai, Science 287, 622 (2000).

3. P.G. Collins, K. Bradley, M. Ishigami, and A. Zettl, Science 287, 1801 (2000).

4. R.J. Baierle, T.M. Schmidt, and A. Fazzio, Solid State Commun. 142, 49 (2007).

5. W. Wang, Y. Zhang, C. Shen, and Y. Chai, AIP Adv. 6, $025317(2016)$

6. W. An, X. Wu, J.L. Yang, and X.C. Zeng, J. Phys. Chem. C 111,14105 (2007).

7. J.-X. Zhao and Y.-H. Ding, J. Chem. Phys. 131, 014706 (2009).

8. S. Peng and K. Cho, Nano Lett. 3, 513 (2003).

9. Y. Xie, Y.-P. Huo, and J.-M. Zhang, Appl. Surf. Sci. 258, 6391 (2012)

10. A. Ahmadi Peyghan, H. Soleymanabadi, and Z. Bagheri, J. Mex. Chem. Soc. 59, 67 (2015).

11. Z. Yong-Hui, C. Ya-Bin, Z. Kai-Ge, L. Cai-Hong, Z. Jing, Z. Hao-Li, and P. Yong, Nanotechnology 20, 185504 (2009).

12. B. Sanyal, O. Eriksson, U. Jansson, and H. Grennberg, Phys. Rev. B 79, 113409 (2009).

13. J. Dai, J. Yuan, and P. Giannozzi, Appl. Phys. Lett. 95, 232105 (2009).

14. A. Saffarzadeh, J. Appl. Phys. 107, 114309 (2010).
15. B. Huang, Z. Li, Z. Liu, G. Zhou, S. Hao, J. Wu, B.-L. Gu, and W. Duan, J. Phys. Chem. C 112, 13442 (2008).

16. R. Chegel, Phys. B Condens. Matter 499, 1 (2016).

17. R. Chegel, Phys. E Low Dimens. Syst. Nanostruct. 84, 223 (2016).

18. Y. Ding, Y. Wang, and J. Ni, Appl. Phys. Lett. 95, 123105 (2009).

19. L. Ci, L. Song, C. Jin, D. Jariwala, D. Wu, Y. Li, A. Srivastava, Z.F. Wang, K. Storr, L. Balicas, F. Liu, and P.M. Ajayan, Nat. Mater. 9, 430 (2010).

20. M. Moradinasab, M. Pourfath, M. Fathipour, and H. Kosina, IEEE Trans. Electron Dev. 62, 593 (2015).

21. Y. Zhou, J. Zhang, C. Ye, X. Miao, and D. Zhang, J. Appl. Phys. 115, 114313 (2014).

22. M. Rostam, M. Yawar, and G. Nader, J. Phys. Condens. Matter 20, 425211 (2008).

23. R. Chegel, Appl. Phys. A 122, 567 (2016).

24. M.P.L. Sancho, J.M.L. Sancho, and J. Rubio, J. Phys. F Met. Phys. 14, 1205 (1984).

25. T.C. Li and S.-P. Lu, Phys. Rev. B 77, 085408 (2008).

26. Y. Imry and R. Landauer, Rev. Mod. Phys. 71, S306 (1999).

27. S. Datta, Quantum Transport: Atom to Transistor (Cambridge: Cambridge University Press, 2005).

28. W.A. Harrison, Electronic structure and the properties of solids (San Francisco: W. H. Freeman and Company, 1980).

29. A. Srivastava, C. Bhat, S.K. Jain, P.K. Mishra, and R. Brajpuriya, J. Mol. Model. 21, 39 (2015).

30. R. Wang and D. Zhang, Aust. J. Chem. 61, 941 (2008).

31. Y.-H. Zhang, K.-G. Zhou, X.-C. Gou, K.-F. Xie, H.-L. Zhang, and Y. Peng, Chem. Phys. Lett. 484, 266 (2010).

32. S. Santucci, S. Picozzi, F. Di Gregorio, L. Lozzi, C. Cantalini, and L. Valentini, J. Chem. Phys. 119, 10904 (2003).

33. N. Maziar, F. Morteza, and P. Mahdi, Jpn. J. Appl. Phys. 51, 035101 (2012).

34. Y. Peng and J. Li, Front Environ. Sci. Eng. 7, 403 (2013).

35. O. Leenaerts, B. Partoens, and F.M. Peeters, Phys. Rev. B 77,125416 (2008). 